\title{
HURRICANE EVACUATION DEMAND MODELS WITH A FOCUS ON USE FOR PREDICTION IN FUTURE EVENTS
}

\author{
Kecheng Xu, Rachel Davidson, Linda Nozick, Tricia Wachtendorf and Sarah E. DeYoung
}

\begin{abstract}
Although substantial literature exists on understanding hurricane evacuation behavior, few studies have developed models that can be used for predicting evacuation rates in future events. For this paper, we develop new ordered probit models for evacuation using survey data collected in the hurricaneprone state of North Carolina in 2011 and 2012. Since all covariates in the models are available from the census or based on location, the new models can be applied to predict evacuation rates for any future hurricane. The out-of-sample predictive power of the new models are evaluated at the individual household level using cross validation, and the aggregated level using available data from Hurricane Irene (2011), Hurricane Isabel (2003) and Hurricane Floyd (1999). Model results are also compared with an existing participation rate model, and a logistic regression model available from the literature. Results at the individual household level suggests approximately $70 \%$ of households' evacuation behavior will be predicted correctly. Errors are evenly divided between false positives and false negatives, and with accuracy increasing to $100 \%$ as the percentage of people who actually evacuate goes to zero or all and decreasing to about $50 \%$ when the population is divided and about half of all households actually evacuate. Aggregate results suggest the new models compare favorably to the available ones, with average aggregate evacuation rate errors of five percentage points.
\end{abstract}

\section{Keywords}

Ordered Probit Model; Hurricane Evacuation Prediction

\section{Introduction}

Empirical and theoretical research on hurricane evacuation behavior has provided a great deal of knowledge about how people make evacuation decisions (Baker, 1991; Dow \& Cutter, 2002; Whitehead et al., 2000). Specifically, prior studies have sought to clarify if, when, how, and to where people evacuate under hurricane threat. That research is of great practical importance because an understanding of the anticipated behavior of affected populations is critical to planning effective and efficient evacuations in future events. Nevertheless, few studies have explicitly discussed use of evacuation behavior research for prediction at the regional level in hypothetical or real future hurricanes. A focus on prediction would require two efforts that, with a few exceptions, have not been part of previous research investigations, even those that developed statistical models with probability of evacuation or similarly relevant response variables. They are: (1) ensuring that all covariates in a statistical model are available for a future event and thus the model can be applied in a predictive mode, and (2) evaluating the out-of-sample predictive power, or ability of the model that was fitted with one sample of data to correctly predict behavior for another.

In this study, we aim to address this gap by developing new statistical models of evacuation behavior with a focus on use in a predictive mode. Specifically, ordered probit models are developed to estimate the number of evacuees in each geographic evacuation zone for a specified future hurricane, with the idea that that information can be used as input into engineering evacuation planning models. The models are developed and tested using survey data from North Carolina.

Section 2 summarizes the literature on quantitative models of evacuation behavior with specific focus on the extent to which they are suitable for use in prediction. In Sections 3 and 4, we describe the data and analysis approach, respectively. The final fitted models are presented in Section 5, and an evaluation of their predictive power at the individual household and aggregate levels are presented in Sections 6 and 7, respectively.

\section{Background}


The literature on evacuation behavior is extensive and many useful reviews are available, such as Dash and Gladwin (2007), Yazici and Ozbay (2008) and Murray-Tuite and Wolshon (2013). It is also worth noting that Russo and Chilà (2014) creates a generic framework for demand modeling in evacuation situations that bridge many types of events including hurricanes. We focus in this paper on studies that relate to quantitative models of evacuation behavior, and specifically consider their suitability for use in prediction. As Wilmot and Mei (2004) discuss, two primary methods have been used to investigate evacuation demand (i.e., probability of evacuation or number of evacuees): (1) participation rates, and (2) regression-type statistical models.

The participation rate approach, which is similar to cross-classification used in conventional transportation planning, is the most commonly used approach in practice, including being used in North Carolina currently (Fu 2004). In this method, participation rates (i.e., percentage of people who will evacuate) are assigned for each geographic region, with different values based on a few key features, such as, hurricane strength and housing type (mobile home or not) (PBS\&J 2002). The product of the population in the affected region and the appropriate participation rate then provides the estimated number of evacuees. Participation rates are relatively coarse, and according to Wilmot and Mei (2004), are typically based on a subjective combination of conclusions from the literature and experience in past storms rather than as output of a particular statistical analysis.

In the second approach, a sample of survey data is used to fit a statistical model in which the household evacuation decision is the response variable and the set of covariates are attributes of the household, hurricane, and/or geographic area. These are typically logit or probit regression models, but include other types as well, and more recently, neural networks. Here we include similar models in which the response variables are related quantities of evacuation timing, or destination type or location. Since our focus is prediction, we consider specifically the extent to which available models may be used in a predictive mode for a future hurricane and/or their predictive power has been evaluated.

Many of the models in the literature were developed with a primary purpose of identifying the variables that are related to the evacuation decision, not being used for prediction. Thus, the final models often include covariates that are not likely to be available for use in prediction (e.g., risk perception), although in some cases, it appears they could be with a relatively small modification (e.g., Riad et al. 1999, Bateman and Edwards 2002, Whitehead 2005, Whitehead et al. 2000, Solis et al. 2009, Solis et al. 2010, Lazo et al. 2010, Petrolia and Bhattacharjee 2010, Hasan et al. 2011, Huang et al. 2012, Hasan et al. 2012, Murray-Tuite et al. 2012, Mesa-Arango et al. 2013, Sadri et al. 2013, Hasan et al. 2013). One of those models did test predictive power with the data they had. Whitehead (2005) included an initial 1999 survey that asked for actual evacuation behavior in Hurricane Bonnie and planned evacuation behavior in future hurricanes, and a follow up 2000 survey of the same respondents that asked if they had actually evacuated during the intervening Hurricanes Dennis and Floyd. The Whitehead study included a computed forecast error as the difference between the predicted probability of evacuation and actual evacuation behavior and found some degree of predictive validity. Since the models include some covariates that would not be available without survey data (e.g., perceived wind risk), however, they still cannot be used directly for prediction in future events. On the other hand, some studies, such as, Cheng et al. (2008), Smith and McCarty (2009), and Reininger et al. (2013) present models that it appears could be used for prediction in a future event, but out-of-sample prediction is not tested or discussed.

Finally, Wilmot and colleagues conducted the only studies we could identify that address prediction directly. Wilmot and Mei (2004) compares five types of evacuation demand modelsparticipation rate, logistic regression, and three types of neural networks - all fitted using $85 \%$ of observations in a 1995 household survey in southwestern Louisiana with respect to Hurricane Andrew. The models' predictive performances were assessed and compared at the aggregate and individual household levels. County level evacuation rates were compared to those observed based on the full dataset. For individual households, predicted and observed behavior was compared using the $15 \%$ of observations withheld for testing. In Section 7, we compare the Wilmot and Mei (2004) logistic regression to the new models developed in this paper. Fu and Wilmot (2006), Fu et al. (2007), and Gudishala and Wilmot $(2012,2013)$ discuss different types of models of time-dependent evacuation demand (i.e., probability a household evacuates in time interval $t$ ). They all discuss prediction explicitly and after using survey data from one hurricane to fit the models, estimate out-of-sample 
predictive accuracy by testing with a $15 \%$ holdout set, with observed data from a different hurricane, or with a different set of stated preference data. Yazici and Ozbay (2008) compare models of timedependent evacuation demand in terms of resulting effects on evacuation clearance and average evacuation time.

\section{Data}

\subsection{Data collection}

Two phone surveys were conducted, creating two datasets for analysis, which we call the 2011 landline and 2012 cell phone datasets. Both surveys were implemented using a computer assisted telephone interviewing (CATI) system, and focused on four areas of North Carolina-Wilmington, Jacksonville, Raleigh, and the Outer Banks - diverse in terms of urban-ness and proximity to coast. In both, only residents were eligible and the surveys lasted approximately 20 minutes on average. Ten thousand phone numbers generated by random digit dialing (RDD) were purchased from Genesys, a third party sample provider. The initial sample of 10,000 numbers was screened to eliminate nonproductive numbers or persons under 18, leaving a total of 3,755 landline numbers and 2,464 cell phone numbers. For the cell numbers, 18 cases were removed from completed interviews because they did not live in the study area (North Carolina). For the landline data, three case of completed interviews were removed due to residing outside of the study area. In the first 2011 landline survey, conducted February to July 2011, the 3,755 landline numbers were called resulting in 284 interviews and an $N_{L}=267$ usable interviews that were complete for all variables included in this particular analysis. The survey included approximately 90 questions, both open-ended and closed format, assessing previous evacuation and sheltering experiences, anticipated future behaviors, explanations of choices, and demographic information. In the 2012 cell phone survey, conducted June to August 2012, 2,446 cell phone numbers were called, resulting in 140 interviews and $N_{C}=137$ complete and usable interviews for the analysis presented in this paper. The survey was the same, with only minor modifications made. Additional analysis related to the landline dataset is reported in [AUTHOR CITATION to be added after review].

There are three important differences between the two surveys: one used landline numbers, the other cell phones; they were conducted a year apart; and in between them, Hurricane Irene made landfall in the Outer Banks of North Carolina as a Category 1 hurricane in August 2011. For the 2011 landline and 2012 cell phone surveys, the mean response rates across the four areas, defined as the number of households that agreed to take the survey divided by the total number of phone numbers left after ineligible numbers were removed (AAPOR 2011), were $10.40 \%$ and $9.23 \%$, landline and cell phone data sets respectively. The mean cooperation rates across the four areas, defined as the number of households that said they would be willing to take the survey divided by the total number of residential numbers interviewers contacted and where a household member agreed or declined to participate (AAPOR 2011), were $16.67 \%$ and $14.18 \%$, landline and cell phone data sets respectively.

\subsection{Data description}

Tables 1, 2, and 3 summarize the descriptive statistics for the 2011 landline and 2012 cell phone datasets for the response, interval, and categorical variables considered in this analysis, respectively. We keep the two datasets separate throughout the analysis because of the differences in how they were collected and because t-tests comparing the variable means showed statistically significant differences in the responses. Respondents in the 2012 cell phone survey were generally younger, more often male, less educated, were more likely to anticipate evacuating and tended to be either working full or part-time than those in the 2011 landline survey (Tables 1-3). All of these differences were statistically significant with $p<0.01$. Given the difference in the responses for the two surveys, we analyzed them separately. This does result in somewhat small sample sizes, especially for the 2012 cell phone survey. However, there since there are two surveys, there is the opportunity to compare the impact of different independent variables across both sets of responses. Further, the goal of this analysis is regionwide prediction and we evaluate performance of the models in multiple ways as described in Section 4.2. 
Respondents were asked "If the local authorities mandated that you evacuate for one of the following conditions, would you actually do so? Please answer yes or no for each." Six conditions were presented-Tropical storm, and Category 1, 2, 3, 4, and 5 hurricanes. They were then asked "If the local authorities issued a voluntary evacuation order for one of the following conditions, would you actually do so? Again, please answer yes or no for each." The same conditions were presented, resulting in twelve responses. We found that all the respondents were rational in that if they said they would evacuate for a category $c$ storm, they also said they would evacuate for all stronger hurricanes as well. Thus, we recoded the twelve responses as shown in Table 1 to obtain the variable "Minimum storm intensity for which the respondent would evacuate given order type (mandatory $y_{m}$, or voluntary $y_{v}$ )," which we used as the ordinal response variables. Note that some of the 12 scenarios presented to the respondents are unlikely to occur (e.g., mandatory order for a tropical storm or voluntary order for a Category 5 hurricane). Nonetheless, those scenarios were presented because they provide an additional check on the consistency of the respondents and they avoided the need to identify threshold storm levels for each type of order and potentially have to explain these threshold levels to the respondents.

These response variables $y_{m}$ and $y_{v}$ can be interpreted as a scale measuring evacuation tendency, with lower values corresponding to higher tendency to anticipate evacuating. Alternatively, it can be used to determine if a respondent anticipated evacuating given a specified hurricane category, as it was in the predictive power analyses (Sections 6 and 7). The location variable values $\left(x_{\text {dist10, }} x_{\text {dist } 10 t o 30}\right)$ were computed in a geographic information system as the straight-line distance to the coast based on address provided. To be consistent with available census data so that the models could be used in a predictive mode (Section 7), age ( $\left.x_{\text {age }}\right)$ was recoded from ordinal values to the nine shown; education $\left(x_{\text {educl }}, x_{\text {educ } 2}\right)$ was recoded from eight categories to the three shown; and number of children was recoded from a number to the binary variable shown. Note that all respondents were eighteen years or older, so there were none in age category 1.

Table 1. Number of responses for each response variable, $y_{m}$ and $y_{v}$, for each dataset

\begin{tabular}{|c|c|c|c|c|c|c|}
\hline & & \multicolumn{2}{|c|}{2011 Landline } & \multicolumn{3}{|c|}{2012 Cell phone } \\
\hline Value & Description & $\begin{array}{c}\text { Mandatory } \\
y_{m}\end{array}$ & $\begin{array}{c}\text { Voluntary } \\
y_{v}\end{array}$ & & $\begin{array}{l}\text { diatory } \\
y_{m}\end{array}$ & $\begin{array}{c}\text { Voluntary } \\
y_{v}\end{array}$ \\
\hline 1 & for $\mathrm{t}$ & $86 \quad(32 \%)$ & $29(11 \%)$ & 49 & $(36 \%)$ & $14(10 \%)$ \\
\hline 2 & nly & $(7 \%)$ & $16 \quad(6 \%)$ & 26 & $(19 \%)$ & $(9 \%)$ \\
\hline 3 & $\mathrm{nly}$ & $20 \quad(7 \%)$ & $29(11 \%)$ & 0 & $(0 \%)$ & $21(15 \%)$ \\
\hline 4 & nly & $42(16 \%)$ & $58(22 \%)$ & 33 & $(24 \%)$ & $44(32 \%)$ \\
\hline 5 & or higher & $55(21 \%)$ & $64(24 \%)$ & 15 & $(11 \%)$ & $19(14 \%)$ \\
\hline 6 & lo & $(7 \%)$ & $(9 \%)$ & 8 & $(6 \%)$ & $(7 \%)$ \\
\hline 7 & Will never evacuate no $\mathrm{n}$ & $(9 \%)$ & $47 \quad(18 \%)$ & 6 & $(4 \%)$ & $17 \quad(12 \%)$ \\
\hline
\end{tabular}

Table 2. Summary statistics for interval variables, for each dataset

\begin{tabular}{c|l|c|c|c|c}
\hline & & \multicolumn{2}{|c|}{ 2011 Landline data } & \multicolumn{2}{c}{ 2012 Cell phone data } \\
\hline Variable & \multicolumn{1}{|c|}{ Variable definition $^{2}$} & Mean & SD & Mean & SD \\
\hline$x_{\text {age }}$ & Age $^{\mathrm{a}}$ & 5.78 & 1.43 & 4.58 & 1.51 \\
$x_{\text {inc }}$ & Annual income $^{\mathrm{b}}$ & 4.80 & 2.72 & 5.31 & 2.74 \\
\hline
\end{tabular}

a Age: $1=$ younger than 15 years old, $2=18-25$ years old, $3=25-35$ years old, $4=35-45$ years old, $5=45-55$ years old, $6=55-65$ years old, $7=65-75$ years old, $8=75-85$ years old, $9=$ older than 85 years old

b Income: $1=$ less than $\$ 20 \mathrm{k}, 2=\$ 20 \mathrm{k}-\$ 30 \mathrm{k}, 3=\$ 30 \mathrm{k}-\$ 40 \mathrm{k}, 4=\$ 40 \mathrm{k}-\$ 50 \mathrm{k}, 5=\$ 50 \mathrm{k}-\$ 60 \mathrm{k}, 6=\$ 60 \mathrm{k}-\$ 70 \mathrm{k}, 7=\$ 70 \mathrm{k}-$ $\$ 80 \mathrm{k}, 8=$ more than $\$ 80 \mathrm{k}$

Table 3. Number (\%) of responses for each categorical variable for each dataset

\begin{tabular}{l|c|c|rc|rr}
\hline Definition & Variable $^{\mathrm{a}}$ & \multicolumn{1}{c|}{ Category } & \multicolumn{2}{|c|}{ 2011 Landline data } & 2012 Cell phone data \\
\hline \multirow{2}{*}{ Location } & $x_{\text {dist10 }}$ & $<10$ miles from coastline & 122 & $(46 \%)$ & 45 & $(33 \%)$ \\
\cline { 3 - 7 } & $x_{\text {dist10to30 }}$ & $10-30$ miles from coastline & 72 & $(27 \%)$ & 31 & $(23 \%)$ \\
\cline { 3 - 6 }
\end{tabular}




\begin{tabular}{|c|c|c|c|c|c|c|}
\hline & - & $>30$ miles from coastline & 73 & $(27 \%)$ & 61 & $(45 \%)$ \\
\hline \multirow{3}{*}{$\begin{array}{l}\text { Housing } \\
\text { type }\end{array}$} & \multirow{3}{*}{$\begin{array}{c}x_{\text {mobile }} \\
x_{S F D} \\
- \\
\end{array}$} & \multirow{3}{*}{$\begin{array}{l}\text { Manufactured or mobile home } \\
\text { Single-family detached home } \\
\text { Other }\end{array}$} & 16 & $(6 \%)$ & 17 & $(12 \%)$ \\
\hline & & & 219 & $(82 \%)$ & 97 & $(71 \%)$ \\
\hline & & & 34 & $(13 \%)$ & 23 & $(17 \%)$ \\
\hline \multirow{2}{*}{ Gender } & \multirow{2}{*}{$x_{\text {male }}$} & \multirow{2}{*}{$\begin{array}{l}\text { Male } \\
\text { Other }\end{array}$} & 101 & $(38 \%)$ & 72 & $(53 \%)$ \\
\hline & & & 166 & $(62 \%)$ & 65 & $(47 \%)$ \\
\hline \multirow{2}{*}{ Race } & \multirow{2}{*}{$x_{\text {white }}$} & \multirow{2}{*}{$\begin{array}{l}\text { White } \\
\text { Other }\end{array}$} & 224 & $(84 \%)$ & 112 & $(82 \%)$ \\
\hline & & & 43 & $(16 \%)$ & 25 & $(18 \%)$ \\
\hline \multirow{3}{*}{ Education } & \multirow{3}{*}{$\begin{array}{c}x_{\text {educ } 1} \\
x_{\text {educ } 2} \\
-\end{array}$} & \multirow{3}{*}{$\begin{array}{l}\text { Less than 2-year college degree } \\
\text { 2- or 4-year college degree } \\
\text { Completed graduate degree }\end{array}$} & 91 & $(34 \%)$ & 55 & $(40 \%)$ \\
\hline & & & 115 & $(43 \%)$ & 60 & $(44 \%)$ \\
\hline & & & 61 & $(23 \%)$ & 22 & $(16 \%)$ \\
\hline \multirow{2}{*}{ Job } & \multirow{2}{*}{$x_{j o b}$} & \multirow{2}{*}{$\begin{array}{l}\text { Has full- or part-time job } \\
\text { Other }\end{array}$} & 131 & $(49 \%)$ & 90 & $(66 \%)$ \\
\hline & & & 136 & $(51 \%)$ & 47 & $(34 \%)$ \\
\hline \multirow{2}{*}{ Child } & \multirow{2}{*}{$\begin{array}{l}x_{\text {child }} \\
-\end{array}$} & \multirow{2}{*}{$\begin{array}{l}\text { Has one or more children } \\
\text { Other }\end{array}$} & 77 & $(29 \%)$ & 54 & $(39 \%)$ \\
\hline & & & 190 & $(71 \%)$ & 83 & $(61 \%)$ \\
\hline
\end{tabular}

a Each variable is a binary variable that is one if in the specified category and zero otherwise

\section{Analysis}

\subsection{Model fitting}

We use ordered probit models of the evacuation decision since the response variables are measured on an ordinal scale as defined in Table 1. That is, the ranking of the values is important (i.e., the lower the value of $y_{m}$ or $y_{v}$, the greater the tendency to evacuate), but the difference between consecutive values is not constant (e.g., the difference between $y_{m}=1$ and $y_{m}=2$ is not necessarily the same as the difference between $y_{m}=2$ and $y_{m}=3$ ). Although the ordered nature can be captured by nested logit and mixed logit models as well, probit models have fewer restrictions than logit models. The ordered probit model more naturally represents ordered alternatives than the logit due to the Independence of Irrelevant Alternatives (IIA) property, which is fundamental to the ordered logit model (Aldrich and Nelson 1984; Hausman and Wise 1978).

The ordered probit model assumes there is a latent continuous variable $y_{i}^{*}$, that describes the exact tendency of household $i$ to evacuate, and thresholds $\alpha_{j}$ divide it into the ordinal categories $j$ that are observed. Specifically:

$$
y_{i}=j \Leftrightarrow \alpha_{j-1}<y_{i}^{*} \leq \alpha_{j}
$$

where $j=1,2, \ldots, 7, \alpha_{0}=-\infty$, and $\alpha_{7}=+\infty$. Further, the latent variable, $y_{i}^{*}$, is a linear combination of covariates:

$$
y_{i}^{*}=\vec{x}_{i}^{\prime} \vec{\beta}+\varepsilon_{i}
$$

where $\vec{x}_{i}^{\prime}$ is a vector of covariates describing attributes of household $i$ or the hurricane under consideration, $\vec{\beta}$ is a vector of coefficients to be estimated by the model, and $\varepsilon_{i} \sim N(0,1)$ is an error term. Note that we assume that neither coefficients $\vec{\beta}$ nor thresholds $\alpha_{j}$ differ over respondents. The probability that household $i$ will take on category $j$ is:

$$
p_{i j}=P\left(y_{i}=j\right)=P\left(\alpha_{j-1}<y_{i}^{*} \leq \alpha_{j}\right)=\Phi\left(\alpha_{j}-\vec{x}_{i}^{\prime} \vec{\beta}\right)-\Phi\left(\alpha_{j-1}-\vec{x}_{i}^{\prime} \vec{\beta}\right)
$$

Simulation-based maximum-likelihood estimation (MLE) procedures are typically used to estimate the parameters in the probit model, and we employ the widely used Geweke-HajivassiliouKeane (GHK) simulator (Geweke 1989; Hajivassiliou and McFadden 1998; Keane 1990, 1994). We use the GHK simulator with MLE in RStudio (version 0.98.953) including package MASS (Venables and Ripley 2002).

We fitted four versions of ordered probit models considering every combination of 2011 landline/2012 cell phone dataset and mandatory/voluntary order. To select the best model in each case we began with all covariates and removed the one with the highest p-value, and refit the model until 
all covariates had $\mathrm{p}<0.1$. It is important to notice that we keep $x_{\text {dist10to30 }}$ with $\mathrm{p}>0.1$ in both mandatory and voluntary 2012 cell phone models. Considering it is close to being significant, we keep $x_{\text {dist10to30 }}$ and we believe that it is an important independent variable as evidenced by the landline models.

\subsection{Model evaluation}

Once the best models were fitted, we evaluated them based on their predictive power at: (1) individual household level, and (2) aggregate level. Since the models are fitted for individual households, it makes sense to evaluate how well they predict individual household behavior. Nevertheless, the models are ultimately intended to be used at an aggregate level (e.g., for counties or larger regions), so that is the level at which prediction should be acceptable. Further, the errors may be smaller at the aggregate level if individual household errors cancel out. That is, within a county, even if the model generates false positives for some households and false negatives for others, the aggregate prediction for the county could still be acceptable.

Predictive power at the individual household level was assessed in two ways (Section 6): (1) tenfold cross validation, and (2) using the models fitted based on the 2012 cell phone data to predict evacuation for the observations from the 2011 landline data, and vice versa. For each of these analyses, we use a model to predict whether particular households will evacuate or not and compare to observations of whether they actually said they would evacuate or not. In each case, we report the four values shown in Table 4.

Table 4. Four error metrics for individual household level prediction

\begin{tabular}{l|c|c}
\hline & Predicted to evacuate & Predicted to not evacuate \\
\hline Actually evacuated & Correct positive & False negative \\
\hline Actually did not evacuate & False positive & Correct negative \\
\hline
\end{tabular}

Predictive power at the aggregate level was assessed as well. For three past hurricanesHurricane Irene (2011), Hurricane Isabel (2003), and Hurricane Floyd (1999), we applied each of our models at the census tract level to estimate the percentage of people who evacuated in each region, and then aggregated up to larger regions to compare to available data on observed evacuation rates and to those that would be predicted by the Wilmot and Mei (2004) logistic regression model and the participation rate model described in the North Carolina 2002 evacuation plan.

\section{Final fitted models}

Table 5 describes the ordered probit model for mandatory and voluntary orders using the 2011 landline and 2012 cell phone data. The models have the likelihood ratio index, also known as McFadden's $R^{2}, \rho^{2}$ values of 0.12 to 0.25 (Table 5), which is consistent with similar models of evacuation behavior, including those in Whitehead (2005), Hasan et al. (2010), Hasan et al. (2013), Mesa-Arango et al. (2013), Fu et al. (2007), Cheng et al. (2008), and Fu and Wilmot (2006), which report $\rho^{2}$ values from 0.10 to 0.32 .

Table 5. Coefficients and p-values of final estimated ordered probit models

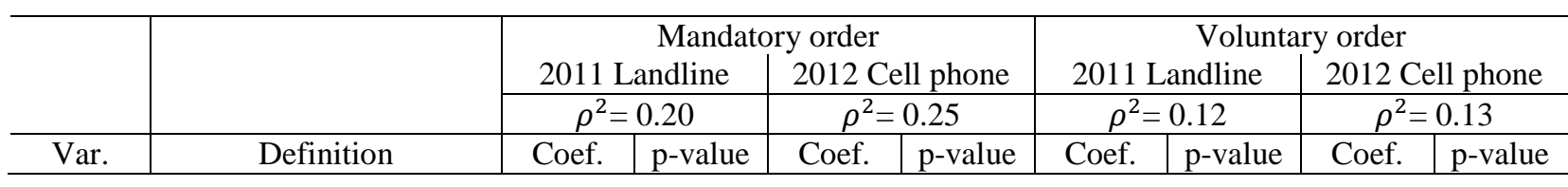




\begin{tabular}{c|l|cc|cc|cc|cc}
$x_{\text {distlo }}$ & $<10$ miles from coast & 1.1232 & 0.0000 & 0.3867 & 0.0856 & 0.6118 & 0.0002 & 0.4789 & 0.0473 \\
$x_{\text {dist10to30 }}$ & 10-30 miles from coast & 0.8756 & 0.0000 & 0.3399 & 0.1793 & 0.5678 & 0.0014 & 0.3259 & 0.1207 \\
$x_{\text {mobile }}$ & Manuf. or mobile home & - & - & - & - & - & - & - & - \\
$x_{\text {SFD }}$ & Single-family detached & - & - & - & - & 0.3109 & 0.0705 & - & - \\
$x_{\text {male }}$ & Male & - & - & 0.4886 & 0.0137 & - & - & 0.3295 & 0.0739 \\
$x_{\text {white }}$ & White & 0.5023 & 0.0126 & - & - & 0.4295 & 0.0214 & 0.5068 & 0.0363 \\
$x_{\text {educl }}$ & $<2-$ year college & - & - & - & - & - & - & - & - \\
$x_{\text {educ } 2}$ & 2- or 4-year college & -0.2727 & 0.0948 & -1.0615 & 0.0009 & - & - & - & - \\
$x_{\text {job }}$ & Full- or part-time job & - & - & - & - & - & - & 0.3656 & 0.0561 \\
$x_{\text {age }}$ & Age & - & - & - & - & - & - & - & - \\
$x_{\text {child }}$ & Children & - & - & - & - & - & - & - & - \\
$x_{\text {inc }}$ & Annual income & - & - & 0.0654 & 0.0724 & - & - & - & - \\
\hline & $1 \mid 2$ & 0.8231 & 0.0001 & 0.7789 & 0.0038 & 0.0191 & 0.9317 & 0.0984 & 0.7202 \\
Thresh- & $2 \mid 3$ & 1.0530 & 0.0000 & - & - & 0.4308 & 0.0572 & 0.6104 & 0.0285 \\
olds, & $3 \mid 4$ & 1.5108 & 0.0000 & 1.5617 & 0.0000 & 1.0585 & 0.0000 & 1.4964 & 0.0000 \\
$\alpha_{j}$ & $4 \mid 5$ & 2.2109 & 0.0000 & 2.0920 & 0.0000 & 1.7244 & 0.0000 & 1.9528 & 0.0000 \\
& $5 \mid 6$ & 2.5860 & 0.0000 & 2.5949 & 0.0000 & 2.0328 & 0.0000 & 2.2824 & 0.0000 \\
\hline
\end{tabular}

a There is no coefficient estimated for $3 \mid 4$ because no one chose alternative " 3 " in 2012 cell phone data, i.e., hence we only estimate 6 alternatives ("1", "2", "4", "5", "6", "7") in 2012 cell phone/mandatory model

A positive sign for a coefficient indicates the associated variable has a negative effect on households' evacuation decisions. For example, the coefficient of $x_{\text {white }}$ in the mandatory/landline model is positive 0.5023 , which means Whites are less likely to anticipate evacuating than nonWhites. In summary, the signs suggest that, given a mandatory or voluntary evacuation order, people are less likely to anticipate evacuating if they live closer to the coast, live in a single-family detached house, are male, are White, did not have 2- or 4-year college degree as their highest educational level, have a full-or part-time job, or have a higher annual income. There have been some mixed findings within the substantial literature on factors affecting evacuation decisions (e.g., Riad et al. 1999). In this study, we did not detect a variable that was surprising in terms of a directionality of effect that had not previously been detected. The one possible exception is our finding that distance to coast is inversely related to likelihood of anticipating evacuation. The effect was robust across the several coding schemes we tried, however. It was also confirmed by direct examination of the underlying data. For the mandatory/landline model, for example, the average values of $y_{m}$ were 4.2, 3.6, and 2.0, for respondents $<10$ miles from the coastline, 10-20 miles from the coastline, and $>30$ miles from the coastline, respectively. The same pattern in which the average minimum storm severity for which respondents say they will evacuate declines as distance from the coastline increases was apparent in the other three models as well.

One possible explanation is that these models are only for cases in which a mandatory or voluntary order has been issued. Not anticipating evacuation, therefore, entails ignoring an official evacuation order, and it may be that households closer to the coast are more likely to do that, perhaps because they have been desensitized by more frequent exposure to hurricanes or for some other reason. In fact, in the survey, we asked people if they had ever ignored an evacuation order before, and we found that in the current datasets, coastal residents are more likely to have ignored an evacuation order in the past than non-coastal residents. In the 2011 landline dataset, only $2.7 \%$ of those living more than 30 miles from the coast have ignored an order before, while $15 \%$ of those 10 to 30 miles from the coast have, and $43 \%$ of those within 10 miles of the coast have. Similarly, in the 2012 cell phone dataset, the percentages are 15\%, 26\% and 27\%, respectively. Within each model, the thresholds $\alpha_{j}$ are reasonably separated, suggesting there is reason to keep the seven-level response variable scale rather than collapsing it into fewer categories.

For each observation in the 2011 landline dataset, we used the mandatory and voluntary order models to compute the probabilities of evacuating given each hurricane category. For each observation and hurricane category, the difference between the resulting probability of evacuating under a mandatory order and probability of evacuating under a voluntary order provided a measure of the effect of the order type, all else being equal. For each hurricane category, the average of the differences over all observations in the dataset offered a summary measure of the effect of order type 
(Table 6). Repeating the analysis for the 2012 cell phone models suggests that overall the change from voluntary to mandatory order result in an increased chance of evacuating of approximately 25 percentage points for storms of Category 2 or weaker and 10 percentage points for storms of Category 3 to 5 .

Table 6. Average probability of evacuation by dataset and order type

\begin{tabular}{l|ccc|ccc}
\hline & \multicolumn{3}{|c|}{ 2011 Landline } & \multicolumn{3}{c}{ 2012 Cell phone } \\
\hline \multicolumn{1}{c|}{ Category } & Mandatory & Voluntary & Difference & Mandatory & Voluntary & Difference \\
\hline Tropical storm & 0.32 & 0.11 & 0.22 & 0.35 & 0.10 & 0.25 \\
Category 1 & 0.39 & 0.17 & 0.23 & 0.54 & 0.19 & 0.35 \\
Category 2 & 0.47 & 0.28 & 0.19 & 0.67 & 0.34 & 0.33 \\
Category 3 & 0.63 & 0.50 & 0.13 & 0.79 & 0.66 & 0.13 \\
Category 4 & 0.83 & 0.74 & 0.10 & 0.90 & 0.80 & 0.10 \\
Category 5 & 0.90 & 0.82 & 0.08 & 0.96 & 0.88 & 0.08 \\
\hline
\end{tabular}

\section{Predictive power at the individual household level}

\subsection{Cross validation}

For each of the four models, we conducted ten-fold cross validation to evaluate predictive power at the individual household. The observations were partitioned into ten randomly-sampled folds. For each fold, the $90 \%$ of observations not in the fold comprised the training set. They were used to fit the models, which were then applied to predict values for each of the $10 \%$ of observations in the foldthe validation set. In this way, we developed out-of-sample predictions of probability of evacuation given hurricane category. To minimize the effect of the particular fold sample, we repeated the cross validation 100 times, each with a different set of randomly-generated folds and averaged the resulting 100 estimates of each error metric for each model.

Figure 1a shows the percentage of out-of-sample observations that were correct (either correctly predicted evacuation or correctly predicted no evacuation), for each model and given each hurricane category. It shows the average correct predictions across all four models and six hurricane categories is $70 \%$ with a range from $52 \%$ to $97 \%$. Neither the 2011 landline or 2012 cell phone models are clearly better when compared with each other, although the latter appear a bit preferred for higher hurricane categories and the former for lower categories. The voluntary models appear to do a better job predicting for lower hurricane categories, while the mandatory models do better for higher categories. As Figure 1b suggests, however, the relative predictive power is really driven by the percentage of people who actually would evacuate. Figure $1 \mathrm{~b}$ shows the percentage of correct predictions vs. percentage of people who said they would evacuate, with an observation for each model and hurricane category. When most people anticipate taking the same action (either evacuate or not), it is easier for the models to predict their behavior correctly. When the population is more divided in their behavior, the models have lower predictive power. This pattern largely explains the differences between the 2011 landline and 2012 cell phone models, and between the mandatory and voluntary models. 

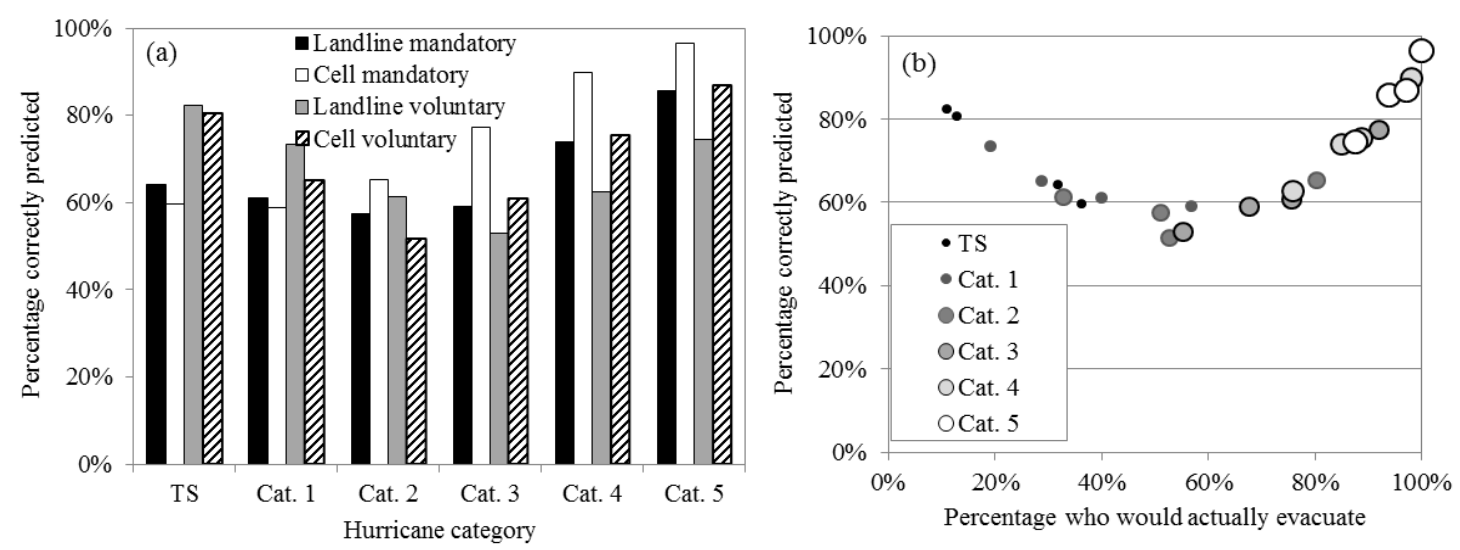

Figure 1. Percentage of correct out-of-sample predictions (a) for each model and hurricane category, and (b) versus percentage of respondents who said they would evacuate

Figure $2 \mathrm{a}$ and $2 \mathrm{~b}$ provide more detailed presentations of the predictions for the 2011 landline models for mandatory and voluntary orders, respectively. They indicate that the incorrect predictions are approximately equally divided between false positives (model predicted evacuation when there actually would not be) and false negatives (model predicted no evacuation when there actually would be). This is promising because it suggests that when the models are applied at an aggregate level (e.g., county), some of those over- and under-predictions may cancel each other out, resulting in an overall evacuation rate that is more accurate. Figures $3 \mathrm{a}$ and $3 \mathrm{~b}$ support similar conclusions for the 2012 cell phone models.

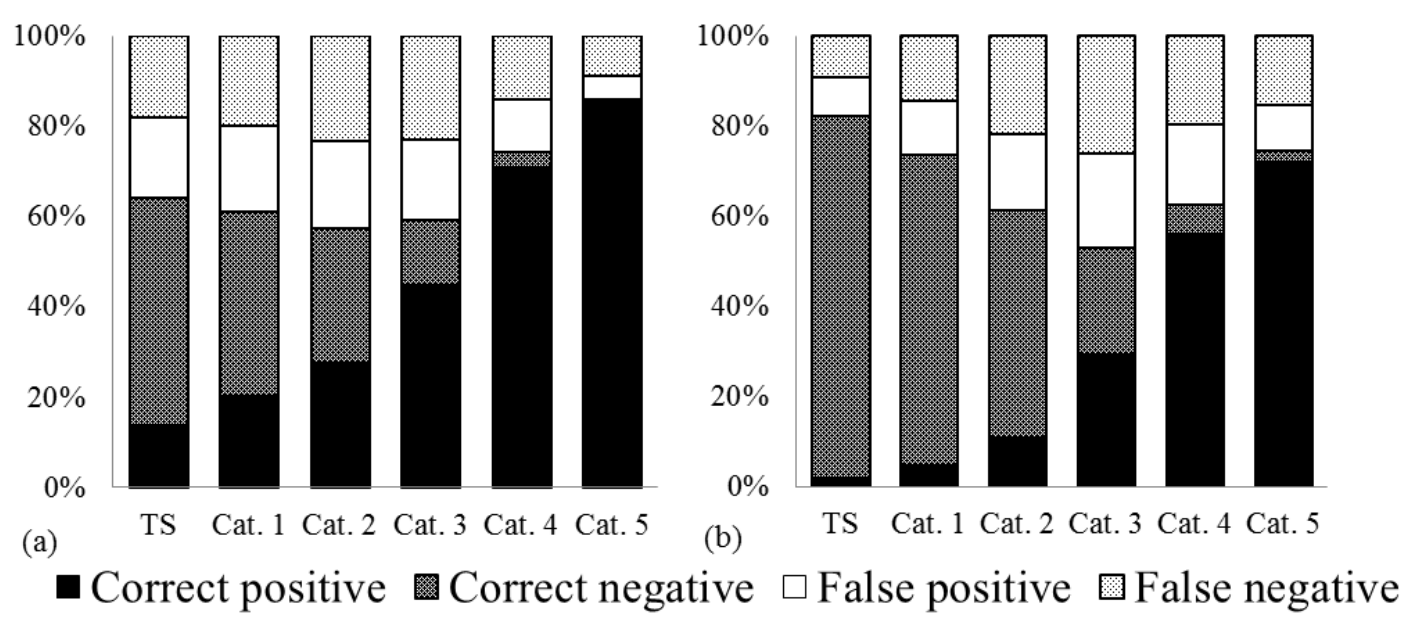

Figure 2. Percentage of correct positive, correct negative, false positive, and false negative predictions using the 2011 landline model for (a) mandatory and (b) voluntary orders, by hurricane category 

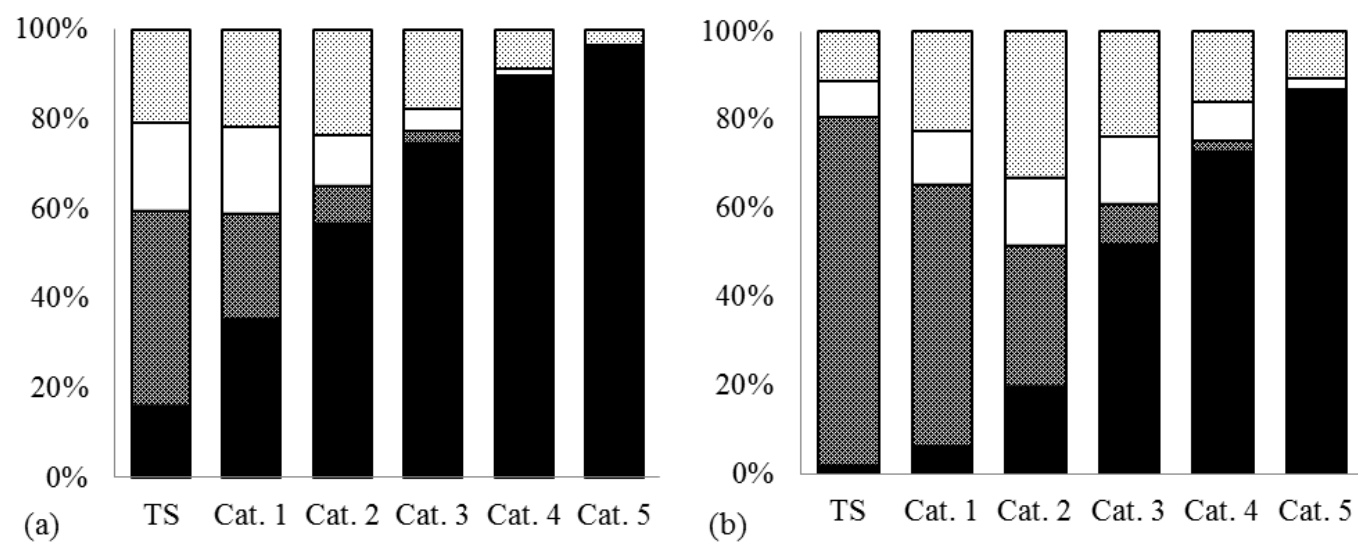

- Correct positive Correct negative $\square$ False positive $\square$ False negative

Figure 3. Percentage of correct positive, correct negative, false positive, and false negative predictions using the 2012 cell phone model for (a) mandatory and (b) voluntary orders, by hurricane category

\subsection{Cross model comparison}

For mandatory orders and then for voluntary orders, we used the model fitted based on the 2012 cell phone data to predict evacuation for the observations from the 2011 landline data, and vice versa. The predictive power and patterns in the results, shown in Figures 4-6, are quite similar to those in the cross validation studies within each dataset (Section 6.1). In particular, averaging across the four models and all hurricane categories, $74 \%$ of predictions were correct, with a range of $53 \%$ to $96 \%$.
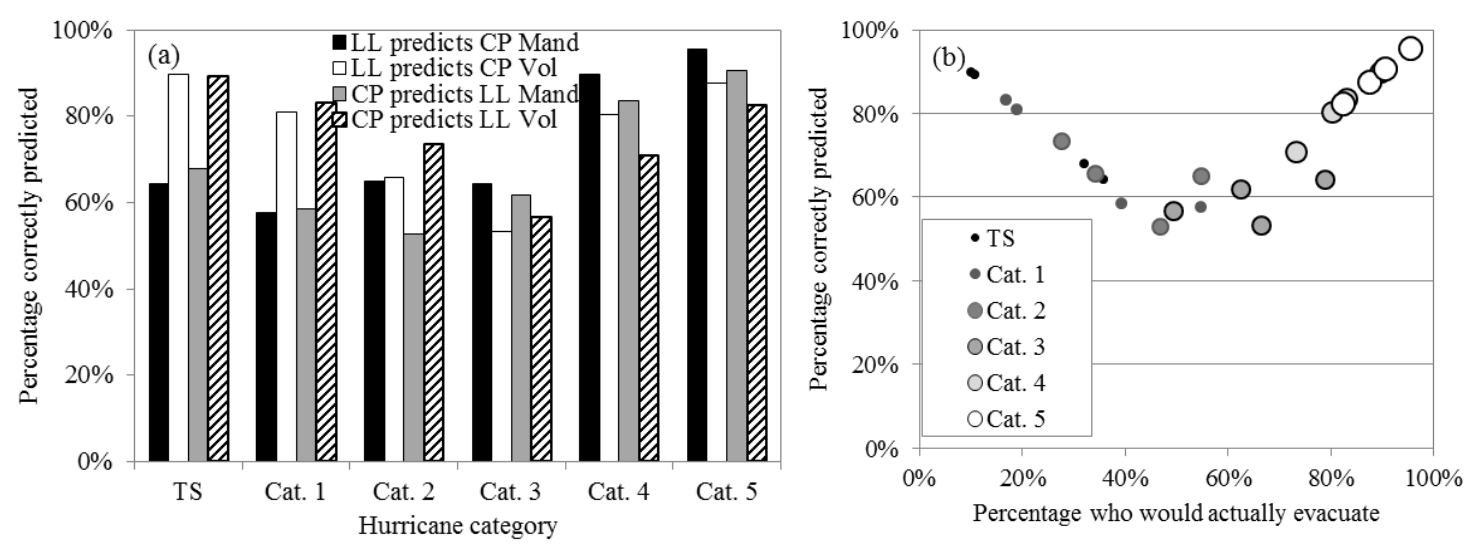

Figure 4. Percentage of correct out-of-sample predictions (a) for each model (LL=landline, $\mathrm{CP}=$ cell phone) and hurricane category, and (b) versus percentage of respondents who said they would evacuate 


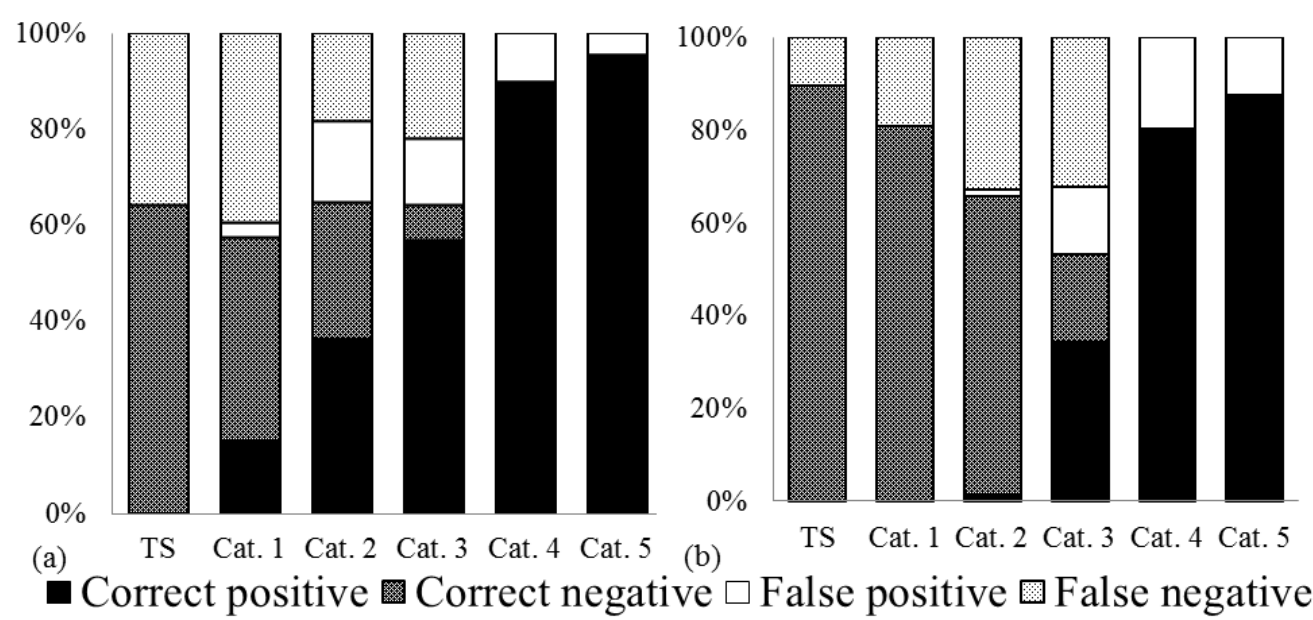

Figure 5. Percentage of correct positive, correct negative, false positive, and false negative predictions using the 2011 landline model to predict 2012 cell phone responses for (a) mandatory and (b) voluntary orders, by hurricane category

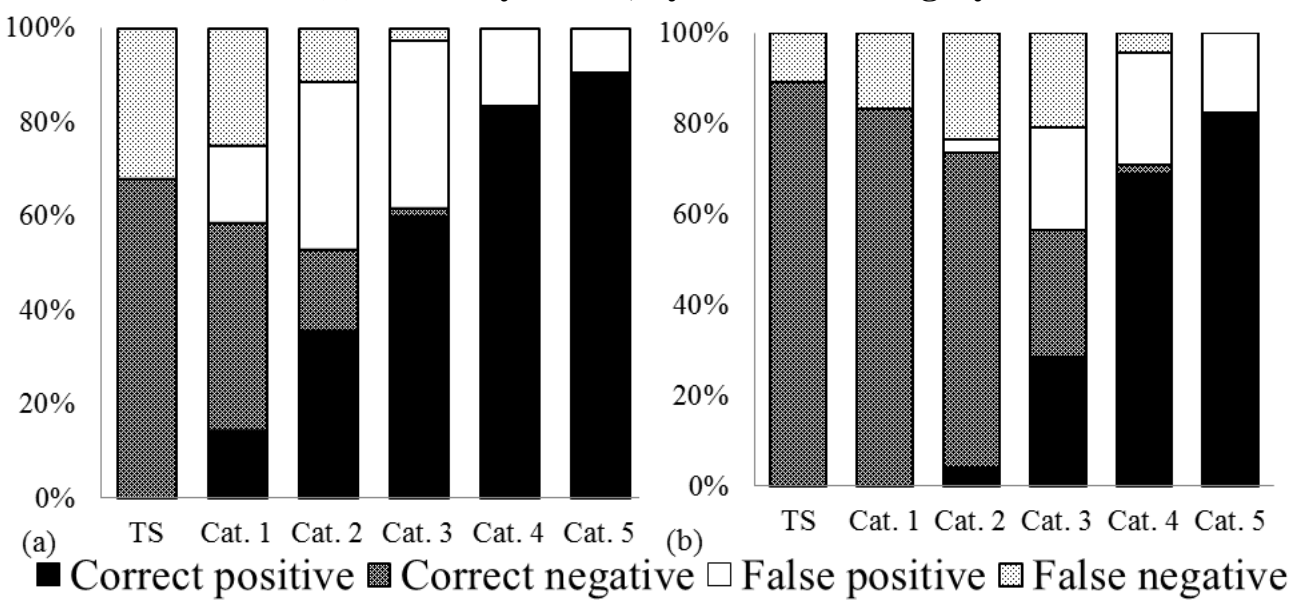

Figure 6. Percentage of correct positive, correct negative, false positive, and false negative predictions using the 2012 cell phone model to predict 2011 landline responses for (a) mandatory and (b) voluntary orders, by hurricane category

\section{Predictive power at the aggregate level}

The second set of tests evaluates the models' predictive power at the aggregate (e.g., county or larger region) level. For three recent hurricanes that affected North Carolina, we compare the ordered probit model predictions to reported evacuation rates and those predicted using the Wilmot and Mei (2004) logistic regression model and the participation rate method currently used in North Carolina (PBS\&J 2002). Survey-based observed evacuation rates in (1) Hurricane Floyd (1999) for Southeastern North Carolina, (2) in Hurricane Isabel (2003) for coastal counties in North Carolina, and (3) in Hurricane Irene (2011) for Beaufort County were reported in PBS\&J (2000), PBS\&J (2005), and Ricchetti-Masterson and Horney (2013), respectively (Figure 7). For Hurricane Isabel, evacuation rates were reported for three regions - Outer Banks North, Outer Banks South, and Sound. For Hurricane Floyd, evacuation rates were available for three regions-Category 1 surge zone, other surge zones, and coastal non-surge zones (non-coastal counties did not have sufficient sample sizes to include in this analysis). In all cases, we used evacuation rates for people who heard the order, since that is consistent with what each of the models represents based on their reported assumptions and how data were collected. Figure 7 includes $90 \%$ confidence intervals on the observed evacuation rates, accounting for the sample size on which they were based. 


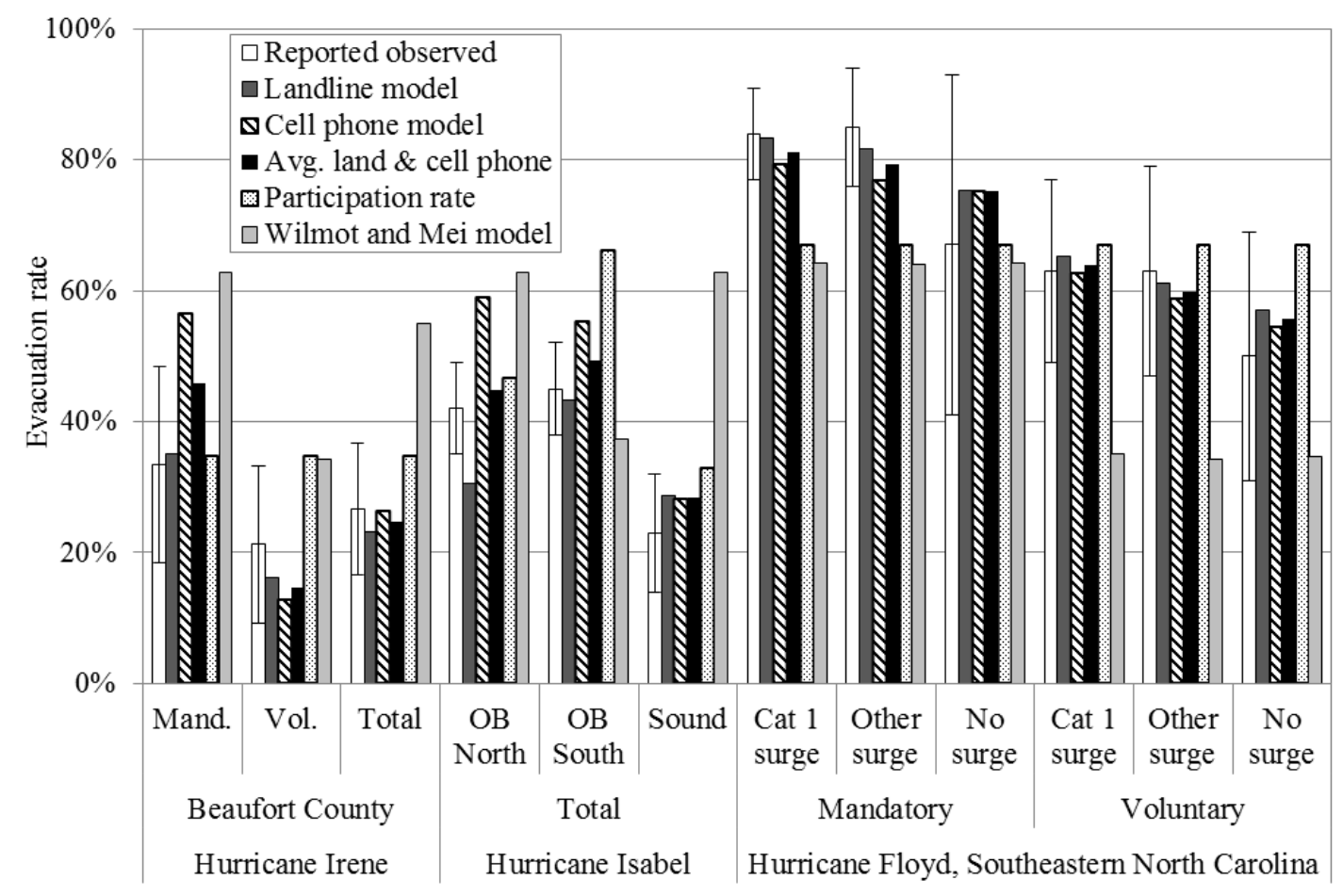

Figure 7. Evacuate rate as reported based on post-event surveys (with $90 \%$ confidence interval) and predicted by different models

To apply one of the ordered probit models to the region, we first applied the model to each census tract separately. That was done by simulating 1000 households for each census tract. The two covariates related to distance to the coast $\left(x_{\text {dist } 10}, x_{\text {dist 10to30 }}\right)$ were based on the shortest "as the crow flies" distance from the population weighted centroid of the tract as given in Centers of Population for Census 2000 (U.S. Census Bureau 2001) to the coast. For all other covariates, the U.S. Census (Social Explorer Professional 2015) provides a distribution of values in each tract. Thus, for each household, we sampled a value for each covariate based on the distribution of values for that covariate in the tract and assuming covariates are uncorrelated. Since all are binary or interval, this involved sampling from discrete distributions. Note that in reality there are correlations among the socio-demographic variables (e.g., income and education), but they were not included here.

We then used the ordered probit model to determine if each simulated household would evacuate or not, and divided by 1000 to compute the tract evacuation rate. The hurricane category was taken to be the hurricane category at the time the order was issued in the county in which that tract is located. The type and timing of evacuation orders were available in Ricchetti-Masterson and Horney (2013), PBS\&J (2005), and PBS\&J (2000). The hurricane categories over time were available in Avila (2011), Beven and Cobb (2004), and Pasch et al. (1999). The evacuation rates for the larger regions used for comparison (e.g., Beaufort County, Outer Banks North) were then computed as the weighted averages of the census tract rates, where the weight for each tract was the percentage of the region population it contains.

Application of the Wilmot and Mei (2004) logistic regression model was conducted similarly. That model predicts a binary choice (i.e., to evacuate or not) as a function of the following covariates, also available in the census: Mobile home, single-family dwelling unit, mandatory evacuation order, age of respondent (years), distance to nearest body of water $\leq 1 \mathrm{mi}$., single and never married, and married or living with partner. If the population-weighted centroid of a census tract is within a mile of the coast, then all households within that census tract were assumed to be within a mile of the coast. This model was calibrated using data collected following landfall of Hurricane Andrew as a Category 3 storm in Louisiana. Hence, implicitly it is calibrated to a Category 3 storm and can be expected to over predict evacuation for lower category events and under predict for higher category events. 
For the participation rate method, evacuation rates are provided in PBS \& J (2002) for each combination of region (Northeastern North Carolina/Southeastern North Carolina), risk area (beaches, other surge, and non-surge for Southeastern NC; outer banks, coastal sound, and non-surge for Northeastern NC), hurricane intensity (strong category 1/strong category 3), and home type (mobile homes/other). To apply the method in these analyses, we selected the appropriate evacuation rates based on location and hurricane intensity. Rates for mobile homes and non-mobile homes were averaged using the percentage of mobile homes in the region as weights.

In total, for each model, there were twelve analyses, one for each combination of hurricane, region, and order type (Fig. 7). The reported evacuation rates range from $21 \%$ to $85 \%$, for the range that is hardest for the ordered probit models to predict (Fig. 1b). The hurricanes were all Category 2 to 3 when evacuation orders were issued in the relevant regions, with the exception of Hurricane Irene, which was a Category 1 hurricane when Beaufort County issued its evacuation order. The comparison in Figure 7 indicate that across all twelve analyses the models result in evacuation rate errors (reported observed - model predicted) of 3 to 16 percentage points on average, depending on the model. To put that in perspective, there are approximately a million people living in the 20 coastal counties of North Carolina. If one-third were under an evacuation order, an error of 3 percentage points would mean an over- or underestimation of 10,000 people.

It is important to note, however, that the reported observed evacuation rates are uncertain given that they are based on surveys of limited sample size. For the 2011 landline model, for example, in only one of the twelve analyses did the error fall outside the $90 \%$ confidence bounds. None of the models has a large bias; they all tend to overestimate in some cases and underestimate in others. With the exception of the participation rate method, the predictions for Hurricane Floyd tended to be the most accurate. The Hurricane Floyd analyses also had the highest evacuation rates. The errors also tend to be smaller for analyses with voluntary orders than for analyses with mandatory orders, which is consistent with the results of the individual household level analysis for hurricanes with lower categories, like those in these analyses.

In comparing the models' predictive powers, the 2011 landline model and the average of the 2011 landline and 2012 cell phone models have the lowest predictive errors on average, with average absolute errors of four and five percentage points, respectively, compared to ten for the participation rate and 21 for the Wilmot and Mei (2004) model. Based on these results, for general application, we recommend using the average of the 2011 landline and 2012 cell phone models. Other considerations when comparing models include the ease and generality of application. The participation rate method is the simplest method to apply, but does not distinguish between different order types and provides a lower level of geographic resolution. The Wilmot and Mei (2004) logistic regression model predictions are not influenced by voluntary orders or hurricane intensity. The ordered probit models developed in this paper are not applicable when no evacuation order is issued.

\section{Conclusions}

In this paper we introduce new ordered probit models that can be used to predict the percentage of households that will evacuate in a future or hypothetical hurricane in each census tract, given a particular order type (mandatory or voluntary). The models are applied in four main steps, as described in Section 7. First, for each census tract in the study region and for each covariate in the models, use the distribution of values recorded in the U.S. Census as an estimate of a discrete probability distribution for the covariate (Table 3). Second, using those discrete distributions for each covariate from Step 1, simulate $N$ (for example, 1000) households for each census tract. Determine if the population-weighted census tract is within 10 miles or is from 10 to 30 miles of the coast. Third, for each simulated household, use the 2011 landline and 2012 cell phone models to predict whether or not the household would evacuate. Assume the hurricane category is that associated with the hurricane at the time the evacuation order is issued. Divide by $N$ to obtain the percentage of households in each census tract that will evacuate for each model. Fourth, average the resulting evacuation rates for the two models to obtain the final estimate for each census tract. If desired, evacuation rates for larger regions can be computed using a population-weighted average of the tract rates. 
Cross validation analysis of predictive power at the individual household level suggests on average about $70 \%$ of households' evacuation behavior will be predicted correctly, with accuracy increasing to $100 \%$ as the percentage of people who actually evacuate goes to zero or all and decreasing to about $50 \%$ when the population is divided and about half of all households actually evacuate. It also indicates that incorrect predictions are evenly divided between false positives and false negatives, so that the errors will cancel each other out to some extent at the aggregate level. Comparisons of the aggregate predictive power across three hurricanes and different regions in Eastern North Carolina suggest errors of five percentage points on average, but up to twelve. They also indicate that in these applications, the new models compare favorably to the method used in current practice in North Carolina and to another model from the literature available for prediction.

Evacuation demand predictions like those provided by these models can inform decisions about when and where emergency managers should issue evacuation orders. They can be incorporated into the decision process either informally as one element under consideration, or formally through their role as input into an engineering evacuation model (e.g., Apivatanagul et al. 2011; Li et al. 2012). Since predictions of evacuation behavior will never be perfect, however, understanding the magnitude and character of the likely error is important as well, again, either to consider informally as part of the process, or formally through a sensitivity analysis with an evacuation model.

Finally, this study suggests a few new directions for future research. The ordered probit models presented apply only when there is a mandatory or voluntary order and the people have heard it. Future work should extend this type of modeling to develop models that estimate the probability of evacuation given no order (or given an order that was not heard) as well as zones in which an order was made. Additional research could also examine more specific attributes of a hurricane and forecast, and their effects on the evacuation decision (e.g., the track or trend in intensity). Finally, hurricane evacuation behavior is a complex decision process that occurs among a heterogeneous population and depends on many variables. To the extent that more data can be collected, models can be improved to capture smaller effects and explain more of the variability in the behavior. Papers such as Dow and Cutter (2002) suggest several considerations of this nature. As more out-of-sample tests of the predictive power of these models are conducted, representing an increasing number and diversity of hurricane events, better assessments of the predictive accuracy will be obtained, ensuring appropriate use of predictions in support of evacuation order decision-making.

\section{Acknowledgments}

This publication was prepared by Cornell University and the University of Delaware using Federal funds under awards 0826832 and 1331269 from the National Science Foundation. The statements, findings, conclusions, and recommendations are those of the authors and do not necessarily reflect the views of the National Science Foundation.

\section{References}

The American Association for Public Opinion Research (AAPOR) (2011). Standard Definitions: Final Dispositions of Case Codes and Outcome Rates for Surveys. $7^{\text {th }}$ edition. AAPOR.

Aldrich, J. H., \& Nelson, F. D. (1984). Linear probability, logit, and probit models (Vol. 45). Sage.

Apivatanagul, P., Davidson, R., Blanton, B., \& Nozick, L. (2011). Long-term regional hurricane hazard analysis for wind and storm surge. Coastal Engineering, 58(6), 499-509.

Avila, L. A. and Cangialosi, J. (2011). Tropical Cyclone Report: Hurricane Irene (AL092011). National Hurricane Center, 45 pp.

Baker, E. J. (1991). Hurricane evacuation behavior. International Journal of Mass Emergencies and Disasters, 9(2), 287-310.Bateman, J. M., \& Edwards, B. (2002). Gender and evacuation: A closer look at why women are more likely to evacuate for hurricanes. Natural Hazards Review, 3(3), 107-117.

Beven, J., \& Cobb, H. (2004). Tropical cyclone report, Hurricane Isabel, 6-19 September 2003. National Oceanic and Atmospheric Association. National Hurricane Center. Miami, Florida. 
Cheng, G., Wilmot, C. G., \& Baker, E. J. (2008). A destination choice model for hurricane evacuation. In Proceedings of the 87th Annual Meeting Transportation Research Board, Washington, DC, USA.

Dash, N., \& Gladwin, H. (2007). Evacuation decision making and behavioral responses: Individual and household. Natural Hazards Review, 8(3), 69-77.Fu, H. (2004). Development of dynamic travel demand models for hurricane evacuation (Doctoral dissertation, Louisiana State University).

Dow K. \& Cutter S.L., (2002). Emerging Hurricane Evacuation Issue: Hurricane Floyd and South Carolina. Natural Hazard Review, 18, 2002.

$\mathrm{Fu}$, H., \& Wilmot, C. G. (2006). Survival analysis-based dynamic travel demand models for hurricane evacuation. Transportation Research Record: Journal of the Transportation Research Board, 1964(1), 211-218.

Fu, H., Wilmot, C. G., Zhang, H., \& Baker, E. J. (2007). Modeling the hurricane evacuation response curve. Transportation Research Record: Journal of the Transportation Research Board, 2022(1), 94-102.

Geweke, J. (1989). Bayesian inference in econometric models using Monte Carlo integration. Econometrica: Journal of the Econometric Society, 1317-1339.

Gudishala, R., \& Wilmot, C. (2012). Comparison of time-dependent sequential logit and nested logit for modeling hurricane evacuation demand.Transportation Research Record: Journal of the Transportation Research Board, 2312(1), 134-140.

Gudishala, R., \& Wilmot, C. (2013). Predictive Quality of a Time-Dependent Sequential Logit Evacuation Demand Model. Transportation Research Record: Journal of the Transportation Research Board, 2376(1), 38-44.

Hajivassiliou, V. A., \& McFadden, D. L. (1998). The method of simulated scores for the estimation of LDV models. Econometrica, 863-896.

Hausman, J. A., \& Wise, D. A. (1978). A conditional probit model for qualitative choice: Discrete decisions recognizing interdependence and heterogeneous preferences. Econometrica: Journal of the Econometric Society, 403-426.

Hasan, S., Mesa-Arango, R., \& Ukkusuri, S. (2013). A random-parameter hazard-based model to understand household evacuation timing behavior.Transportation research part C: emerging technologies, 27, 108-116.

Hasan, S., Mesa-Arango, R., Ukkusuri, S., \& Murray-Tuite, P. (2012). Transferability of hurricane evacuation choice model: Joint model estimation combining multiple data sources. Journal of transportation engineering, 138(5), 548-556.

Hasan, S., Ukkusuri, S., Gladwin, H., \& Murray-Tuite, P. (2010). Behavioral model to understand household-level hurricane evacuation decision making.Journal of Transportation Engineering, 137(5), 341-348.

Huang, S. K., Lindell, M. K., Prater, C. S., Wu, H. C., \& Siebeneck, L. K. (2012). Household evacuation decision making in response to Hurricane Ike.Natural Hazards Review, 13(4), 283296.

Keane, M. P. (1990). Four essays in empirical macro and labor economics. Publisher not identified.

Keane, M. P., \& Wolpin, K. I. (1994). The solution and estimation of discrete choice dynamic programming models by simulation and interpolation: Monte Carlo evidence. The Review of Economics and Statistics, 648-672.

Lazo, J. K., Waldman, D. M., Morrow, B. H., \& Thacher, J. (2010). Household evacuation decision making and the benefits of improved hurricane forecasting: Developing a framework for assessment. Weather and Forecasting, 25, 207.

Li, A., Nozick, L., Xu, N, and Davidson, R. (2012). Shelter Location and Transportation Planning Under Hurricane Conditions. Transportation Research Part E, 48(4), 715-729.

Mesa-Arango, R., Hasan, S., Ukkusuri, S. V., \& Murray-Tuite, P. (2013). Household-level model for hurricane evacuation destination type choice using hurricane Ivan data. Natural hazards review, 14(1), 11-20.

Murray-Tuite, P., \& Wolshon, B. (2013). Evacuation transportation modeling: An overview of research, development, and practice. Transportation Research Part C: Emerging Technologies, 27, 25-45. 
Murray-Tuite, P., Yin, W., Ukkusuri, S. V., \& Gladwin, H. (2012). Changes in evacuation decisions between Hurricanes Ivan and Katrina. Transportation Research Record: Journal of the Transportation Research Board, 2312(1), 98-107.

Pasch, R. J., Kimberlain, T. B., \& Stewart, S. R. (1999). Preliminary report, Hurricane Floyd 7-17 September 1999. Silver Spring, MD: National Oceanic and Atmospheric Administration, National Hurricane Center. Accessed May 14, 2015.

Retrieved from: http://www.nhc.noaa.gov/1999floyd.html.

Petrolia, D., \& Bhattacharjee, S. (2010). Why don't coastal residents choose to evacuate for hurricanes? Coastal Management, 38, 97-112.

PBS\&J. (2000). Southeast United States Hurricane Evacuation Traffic Study: Executive Summary. Prepared for the Federal Emergency Management Agency and U.S. Department of Transportation, Washington, D.C..

PBS\&J. (2002). North Carolina Hurricane Evacuation Restudy: Technical Data Report. Prepared for the Federal Emergency Management Agency, U.S. Army Corps of Engineers, National Oceanic and Atmospheric Administration, and North Carolina Division of Emergency Management, Washington, D.C..

PBS\&J. (2005). Hurricane Isabel Assessment: Review of hurricane evacuation study products and other aspects of the National Hurricane Mitigation and Preparedness Program (NHMPP) in the context of the Hurricane Isabel Response.

Reininger, B. M., Raja, S. A., Carrasco, A. S., Chen, Z., Adams, B., McCormick, J., \& Rahbar, M. H. (2013). Intention to comply with mandatory hurricane evacuation orders among persons living along a coastal area. Disaster medicine and public health preparedness, 7(01), 46-54.

Riad, J., Norris, F., \& Ruback, R. (1999). Predicting evacuation in two major disasters: Risk perception, social influence, and access to resources. Journal of Applied Social Psychology, 29(5), 918-934.

Ricchetti-Masterson, K., \& Horney, J. (2013). Social factors as modifiers of Hurricane Irene evacuation behavior in Beaufort County, NC. PLoS currents, 5.

Russo, F., \& Chilà, G. (2014). Integrated travel demand models for evacuations: a bridge between social science and engineering. International Journal of Safety and Security, 4(1), 19-37.

Sadri, A. M., Ukkusuri, S. V., Murray-Tuite, P., \& Gladwin, H. (2013). How to evacuate: model for understanding the routing strategies during hurricane evacuation. Journal of transportation engineering, 140(1), 61-69.

Smith, S. K., \& McCarty, C. (2009). Fleeing the storm (s): An examination of evacuation behavior during Florida's 2004 hurricane season. Demography, 46(1), 127-145.

Social Explorer Professional. "2000 Census Tract - North Carolina.” Table. Social Explorer, 2015. Accessed May 14, 2015.

Retrieved from: http://www.socialexplorer.com/.

Solís, D., Thomas, M., \& Letson, D. (209). Determinants of Household Hurricane Evacuation Choice in Florida. Presentation at the Southern Agricultural Economics Association Annual Meeting, Atlanta, Georgia, January 31-February 3, 2009.Solís, D., Thomas, M., \& Letson, D. (2010). An empirical evaluation of the determinants of household hurricane evacuation choice. Journal of Development and Agricultural Economics, 2(3), 188-196.

U.S. Census Bureau (2001). Centers of Population for Census 2000. Accessed May 14, 2015. Retrieved from: https://www.census.gov/geo/reference/centersofpop2000.html.

Venables, W. N., \& Ripley, B. D. (2002) Modern Applied Statistics with S. Fourth Edition. Springer, New York. ISBN 0-387-95457-0.

Whitehead, J. C. (2005). Environmental risk and averting behavior: Predictive validity of jointly estimated revealed and stated behavior data. Environmental and Resource Economics, 32(3), 301316.

Whitehead, J. C., Edwards, B., Van Willigen, M., Maiolo, J. R., Wilson, K., \& Smith, K. T. (2000). Heading for higher ground: factors affecting real and hypothetical hurricane evacuation behavior. Global Environmental Change Part B: Environmental Hazards, 2(4), 133-142.

Wilmot, C. G., \& Mei, B. (2004). Comparison of alternative trip generation models for hurricane evacuation. Natural hazards review, 5(4), 170-178. 
Yazici, M. A., \& Ozbay, K. (2008). Evacuation modelling in the United States: does the demand model choice matter?. Transport Reviews, 28(6), 757-779. 